LIAMES 11 - pp. 51-74, Primavera 2011

Javier J. Carol

(Universidad de Buenos Aires, Argentina)

\title{
Aplicativos/adposiciones en chorote (mataguayo): algunos aspectos formales"
}

\begin{abstract}
In Chorote, certain morphemes which typically introduce an argument in oblique case may attach to the verb (as applicatives) or to the noun (as adpositions), or even be free morphemes. This paper examines some formal issues regarding these morphemes, in particular their order relative to other words and their underlying syntactic structure.

Among these morphemes, polysyllabic ones may occur as postpositions or independent words, since under certain conditions they need not be adjacent to the NP/DP. When this is the case, they take a null pronominal complement which is doubled by the NP/DP. Monosyllabic ones, on the other hand, must attach either to the noun or to the verb. Thus, I claim that applicatives in chorote are underlyingly monosyllabic adpositions -or adpositions plus a null pronominal complement- which encliticize to the verb when they cannot attach to their lexical complement. This phenomenon differs from incorporation of an adposition to the verb. On the other hand, the opposition between adpositional complements and applied arguments does not correspond to that between arguments and adjuncts. Finally, the paper briefly surveys cases where applicatives/adpositions do not seem to add a new argument, at least explicitly.
\end{abstract}

KEYWORDS: Applicatives; Adpositions; Chorote; Mataguayo; Matakoan.

RESUMEN: En chorote, ciertos morfemas que típicamente introducen un argumento en caso oblicuo pueden ligarse al verbo (aplicativos) o al nombre (adposición), o incluso ser morfemas libres. El presente artículo aborda algunos aspectos formales de ellos, particularmente el orden de palabras y las estructuras sintácticas subyacentes que reflejan.

* La investigación realizada para el presente trabajo se financió parcialmente mediante una beca de la Agencia Nacional de Promoción Científica y Tecnológica (ANPCyT) en el marco del PICT 32894 Clasificación nominal y categorización etnobiológica en grupos indígenas del Gran Chaco, y mediante el proyecto UBACyT F444: Lenguas indígenas del Gran Chaco (toba, toba-pilagá, maká y chorote). Agradezco a los miembros de dichos equipos por sus aportes y su apoyo. Quisiera agradecer también a quienes compartieron conmigo sus conocimientos sobre su lengua chorote, especialmente a Víctor Díaz, Héctor Sarmiento, Julián Gómez, Nicasio Carrizo, Juan González, Sebastián Frías y Roberto Valentín. Agradezco también a Cristina Messineo, Andrés Salanova, Alain Fabre y Alicia Avellana por comentarios, discusión y sugerencias.

Los datos utilizados en el artículo provienen de trabajo de campo realizado por el autor entre 2005 y 2010, de los vocabularios de Drayson $(1999,2009)$, de relatos recopilados en Drayson et al. (2000) y de la traducción de la Biblia al chorote iyojwa'aja' (AA. VV. 1997). Éstos se abreviarán de la siguiente manera: 500 (Drayson 1999), Sake' (Drayson et al. 2000), DICC (Drayson 2009) y la cita correspondiente en la traducción de la Biblia. Los ejemplos tomados de vocabularios se citarán por la entrada léxica, p. ej. "Dicc: 'YiтA'м". 
De estos morfemas, los polisilábicos pueden ocurrir como posposiciones o constituir palabras independientes, ya que bajo ciertas condiciones no necesitan ser adyacentes al SN/SD; en este caso, toman un complemento pronominal nulo que resulta doblado por el SN/SD. Los monosilábicos, en cambio, deben ligarse obligatoriamente, sea al nombre o al verbo. Postulo que los aplicativos en chorote son adposiciones subyacentes -o adposiciones más un complemento pronominal nulo- que, cuando no pueden ligarse a su complemento léxico, se encliticizan al verbo. Este fenómeno difiere de la incorporación de la adposición al verbo. Por otro lado, la distinción entre complementos adposicionales y argumentos aplicados no se corresponde con una distinción entre argumentos y adjuntos. Por último, el artículo examina brevemente algunos casos en los que los aplicativos/adposiciones no parecen añadir un nuevo argumento, al menos explícitamente.

PALABRAS CLAVES: Aplicativos; Adposiciones; Chorote; Mataguayo.

\section{INTRODUCCIÓN}

En chorote, ${ }^{1}$ ciertos morfemas que típicamente introducen un argumento en caso oblicuo pueden ocurrir ligados al verbo (denominados entonces "aplicativos" (1)) o al nombre ("adposiciones", (2)), e incluso algunos de ellos pueden ser morfemas libres (3) según condicionamientos morfofonológicos y sintácticos. Llamaré P a estos morfemas. $^{2}$

${ }^{1}$ El chorote se habla en el Chaco argentino y paraguayo, y pertenece a la familia mataguaya, junto con el wichí (mataco), nivaclé (chulupí) y maká. La familia es denominada a menudo matakoan en inglés, si bien "mataco" es peyorativo y debe evitarse. El número de sus hablantes posiblemente no supere los 3000, de los cuales la mayor parte habita en territorio argentino. El presente estudio se centra en la variedad iyojwa'aja' (o iyojwa'ja), hablada exclusivamente en Argentina. Estudios descriptivos previos fueron realizados por Gerzenstein $(1978,1979,1983)$.

${ }^{2}$ La grafía adoptada se basa en la que se encuentra en uso en comunidades chorotes de la Argentina, la cual a su vez se basa en la que fue acordada por las comunidades en 1981 (Drayson 2009: 92). Esta grafía se aparta de la del español en lo siguiente: <'> (apóstrofo) indica una oclusión glotal o glotalización de la consonante adyacente; $\langle j\rangle$ es una fricativa laríngea $[\mathrm{h}]$ o velar $[\mathrm{x}] ;\langle s\rangle$ puede articularse $[\mathrm{hs}, \mathrm{hx}]$ en contexto intervocálico; $\langle w, y\rangle$ son siempre semivocales. Sin embargo, me he apartado de la grafía en uso en lo siguiente: $\langle$ e,$o\rangle$ representan vocales altas muy abiertas $[\mathrm{l} v]$ o medias cerradas $[\mathrm{e} o]$, en contraste con $\langle e, o\rangle$, que son medias abiertas [ $\varepsilon$ o] (la grafía en uso no hace esta distinción), y las secuencias de consonante más semivocal palatal son representadas aquí como $\langle C y\rangle$ y no como $\langle C i\rangle$. Además, uso tilde siempre que el acento de intensidad no recae sobre la primera sílaba de la raíz.

Abreviaturas: 1, 2, 3: primera, segunda y tercera personas; A: sujeto de verbo transitivo; $\mathrm{A}^{+}$: sujeto de verbo divalente (A o S); BEn: benefactivo; caus: causativo; com: comitativo; сомp: complementante; cons: conjetural; $\mathrm{D}_{1}-\mathrm{D}_{6}$ : determinante demostrativo (1:ostensible, 2:cercano, 3:distante, 4:alejándose/muerto/desaparecido, 5:no visible, 6:inaccesible/desconocido); DIST: distal; DISTR: distributivo; f: femenino; FOC: focalizador; FPROx: futuro próximo; h: humano; IMPRS: impersonal; inst: instrumental; IPFV: imperfectivo; IRR: irrealis; ITER: iterativo/habitual; JEN: clítico jen (plural verbal; 'hacia abajo'); $K$ : sufijo - $k$ (información vieja, ¿adverbializador, pronominalizador?), $K A$ : sufijo - $k a$ (¿pronominalizador ?); LOC.D: locativo 'dentro de'; Loc.G: locativo general; Loc.P locativo puntual; NEG: negación; nh: no humano; NMZ: nominalizador; O: objeto de verbo transitivo; OR: orientación; P: aplicativo/adposición; PAH: participante del acto de habla; PFv: perfectivo; PL/pl: plural; POS: poseedor; POS.IND: poseedor indefinido; PREM: pasado remoto; PRSP: prospectivo; QU: constituyente interrogativo; S: sujeto de verbo intransitivo; $\mathrm{S}_{\mathrm{A}}, \mathrm{S}_{\mathrm{O}}$ : $\mathrm{S}$ marcado como $\mathrm{A}, \mathrm{O} ; \mathrm{S}$ : sujeto de verbo monovalente; sg: singular; $v$ : verbo liviano. 
Carol: Aplicativos/adposiciones en Chorote (mataguayo): algunos...

(1)

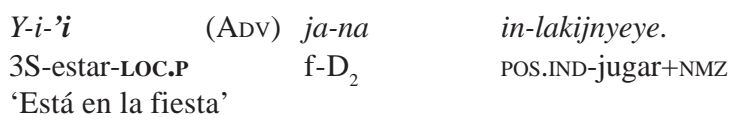

(2)
$Y-i$
(ADv) in-kijmaye
'wet-'e'.
3S-estar POS.IND-trabajar+NMZ
[3POS]lugar-LOC.P

'Está en el ingenio (lit. 'lugar de trabajo')'

(3)

$\begin{array}{llll}Y-i & (\mathrm{ADV}) & \text { apé'e (ADV) ' } n i & \text { ajnát. } \\ \text { 3S-estar } & \text { sobre } \quad \mathrm{D}_{1} & \text { tierra } \\ \text { 'Está sobre la tierra.' (Es decir, 'existe') } & \end{array}$

Los PP polisilábicos pueden ocurrir como posposiciones o constituir palabras fonológica y morfológicamente independientes, como en (3), donde apé'e es independiente fonológicamente (tiene su propio acento de intensidad) y también morfológicamente, ya que allí no necesita ser adyacente a su complemento; en este caso, considero que toma un complemento pronominal nulo que resulta doblado por su "complemento" léxico. Los PP monosilábicos, en cambio, deben ligarse obligatoriamente en vista de su condición fonológicamente defectiva, cf. (1)-(2). Postulo entonces que los aplicativos en chorote son adposiciones subyacentes -o adposiciones más un complemento pronominal- que, cuando no pueden ligarse a su complemento léxico por razones sintácticas que se examinarán aquí, se encliticizan al verbo. Nótese que este fenómeno es superficial y diferente de la incorporación de una adposición al verbo, propuesta en Baker (1988) como origen de los aplicativos.

Por otro lado, la distinción entre complementos adposicionales y argumentos aplicados -los argumentos introducidos por el aplicativo- no se corresponde en chorote con una distinción entre argumentos y adjuntos, ya que sintagmas claramente argumentales pueden ocurrir como complementos adposicionales y quizá también, aunque más raramente, sintagmas prima facie adjuntos pueden ocurrir como argumentos aplicados. Tampoco se corresponde con una distinción entre argumentos oblicuos y directos ya que, según se ha expresado, los argumentos de $\mathrm{P}$ son marcados en cualquier caso como oblicuos.

Como se desprende de lo anterior, el término "aplicativo" se utiliza aquí en un sentido algo diferente del habitual, por lo cual conviene definirlo antes de abordar el análisis. Nótese ante todo que el fenómeno aquí descripto es bastante inusual y que no resulta sencillo hallar un rótulo estándar para definirlo; los términos "direccional" o "locativo" no serían adecuados, ya que $\mathrm{P}$ introduce regularmente un argumento y éste no es necesariamente locativo. Los aplicativos del chorote, pues, no son aplicativos canónicos como los de, por ejemplo, las lenguas bantúes, ya que no implican un "incremento de valencia" en relación con su contraparte adposicional, ni tampoco vuelven transitivo un verbo intransitivo, ya que el argumento regido es un oblicuo. Con todo, sí afectan la valencia o adicidad del predicado en relación con una contraparte sin $\mathrm{P}$ cuando el complemento introducido es argumental, cf. nam 'viene' y nam-e 'trae', lit 'viene con'. En efecto, $\mathrm{P}$ forma aquí un predicado complejo con el verbo $\mathrm{y}$, en consecuencia, añade un nuevo argumento al predicado, si bien no al núcleo de la predicación, esto es, al verbo. En base a esto, entonces, es que propongo llamar "aplicativo" a P cuando se liga al verbo, en 
cuanto es allí un morfema ligado al verbo que introduce un argumento en el predicado (véase Dourado $(2000,2004)$ sobre un fenómeno relativamente similar en panará (jê) y sobre un uso análogo del término "aplicativo"). ${ }^{3}$

El resto del artículo se organiza de la siguiente manera. La sección 2 lista y caracteriza semánticamente - en forma sucinta- las PP. La sección 3 analiza el orden en que ocurre P en la cláusula: cuándo debe ligarse y a qué; también expone el orden relativo en que ocurren los aplicativos. La sección 4 caracteriza morfológica y semánticamente los complementos que puede tomar $\mathrm{P}$ según sea aplicativo o adposición. La sección 5 discute el status formal de $\mathrm{P}$ y su complemento, argumentándose que, cuando P es aplicativo, no introduce un argumento central sino oblicuo y que difiere de un aplicativo canónico. La sección 6 se dedica a algunos casos en los que P no añade un argumento más al predicado, al menos de modo explícito. Finalmente, se presentan las conclusiones.

\section{MORFOSINTAXIS GENERAL: INVENTARIODE PP}

Para comprender la morfosintaxis de los aplicativos/adposiciones convendrá distinguir entre monosilábicos y polisilábicos. Los primeros se ligan obligatoriamente, sea al nombre o al verbo, mientras que los segundos pueden ser palabras fonológica y morfológicamente independientes. También pueden distinguirse clases según la forma del pronombre personal que tomen como complemento cuando éste corresponde a un participante del acto de habla (PAH; en tercera persona el pronombre es normalmente cero): un grupo toma personales que son isomórficos con -o que incluyen a- los posesivos en todas las personas, otro grupo toma personales diferentes de los posesivos (salvo en la segunda persona) y un tercer grupo no toma marcas de persona como complemento. En relación con algunas PP, cabe conjeturar que se originaron en un SP cuyo complemento nominal quedó fosilizado (véase la sección 3).

La TABLA 1 lista las PP que existen en chorote. En vista del isomorfismo de algunas de ellas en numerosos contextos, se exponen también las formas subyacentes. Los procesos morfofonológicos que tienen lugar en chorote iyojwa'aja' son numerosos y complejos, y muy a menudo oscurecen la relación entre las formas superficiales y subyacentes; los principales de ellos se explican en el ApÉNDICE al final del presente artículo.

${ }^{3}$ El término "aplicativo" se ha usado en las últimas décadas para nombrar fenómenos igualmente divergentes de la aplicativización canónica. Así, por ejemplo, en lingüística formal la noción de construcción aplicativa se ha extendido a ciertos objetos indirectos que, al menos en su forma superficial, son encabezados por preposiciones, como en el caso de ciertos dativos del hebreo y de las lenguas romances (Pylkkänen 2002; véase Masullo (1992) y Pujalte (2009) para el español) y a argumentos introducidos por un "aplicativo cero" (Marantz 1984; cf. en inglés I baked-Ø John a cake, en español 'Le cociné una torta a Juan'). En vista de esto, no veo razones para no extender el término al fenómeno aquí tratado, máxime en vista de que las alternativas no son mejores. Por otro lado, nótese que, mientras los "aplicativos" según se los entiende en los estudios formales mencionados poseen muchas de las características de los aplicativos canónicos excepto las morfológicas (no implican necesariamente un morfema manifiesto ligado al verbo), los que llamo "aplicativos" en chorote implican, justamente, un morfema ligado al verbo, aunque no algunas de las demás características prototípicas. 
TABla 1: aplicativos/adposiciones (PP)

\begin{tabular}{|c|c|c|c|}
\hline \multirow{4}{*}{$\begin{array}{l}\text { pro } \\
\neq \\
\text { POS } \\
\text { (exc. } 2^{\mathrm{a}} \text { pers) }\end{array}$} & $-e j \quad /-\mathrm{eh} /$ & instrumental; tema; sociativo & INST \\
\hline & $-e \quad /-\mathrm{ey}^{\mathrm{h}} /$ & distal, 'a/de'; propósito & DIST \\
\hline & -jam /-ham/ & locativo general; dativo & LOC.G \\
\hline & $-a j \quad /-\mathrm{ah} /$ & meta/origen figurado, orientación & OR \\
\hline \multirow{3}{*}{ sin pro } & $-' e \quad /-1 \mathrm{e} /$ & locativo puntual & LOC.P \\
\hline & $-j i \quad /-h i(y) /$ & 'dentro de' & LOC.D \\
\hline & kisye'e ¿/k ${ }^{\mathrm{y}} \mathrm{i}^{\prime} \mathrm{sa}: / ?$ & 'fuera (de)' & fuera \\
\hline \multirow{7}{*}{$\begin{array}{l}\text { pro } \\
= \\
\text { POS }\end{array}$} & \multirow{2}{*}{$-k^{\prime} i \quad /-\mathrm{k}^{\mathrm{y}}{ }^{\prime} \mathrm{e} /$} & 'a lo largo de, a través de’; distributivo & DISTR \\
\hline & & comitativo & $\mathrm{COM}$ \\
\hline & -jap $/ \mathrm{h} \alpha \mathrm{p} /$ & 'junto a'; comitativo & junto.a \\
\hline & apée / / 'pe?e/ & 'sobre'; 'acerca de' & sobre \\
\hline & k'iyé / $/ \mathrm{k}^{\mathrm{y}}$ o'yah/ & $\begin{array}{l}\text { benefactivo; 'en dirección a', 'en relación } \\
\text { con' }\end{array}$ & BEN \\
\hline & ilyá'm /i'l(y)am/ & 'viniendo' & viniendo \\
\hline & kyajwé $/ \mathrm{k}^{\mathrm{y}} \alpha^{\prime} \mathrm{hwe}: \mathrm{h} /$ & 'debajo de', 'dentro de' & bajo \\
\hline
\end{tabular}

\subsection{Algunos comentarios sobre la fonología y morfología}

A causa de diversos procesos de asimilación vocálica progresiva y regresiva a través de laríngeas (véase APÉNDICE), algunas PP resultan homófonas en determinados contextos; esto sucede con el intrumental/tema/sociativo - ej y el distal $-e,{ }^{4} \mathrm{e}$ incluso $-a j$ ' $m e t a / o r i g e n$ figurado, orientación' puede confundirse con ellos. Por ejemplo, dada la secuencia - $a j-\boldsymbol{a}\left(1 \mathrm{plA} / \mathrm{S}_{\mathrm{A}}-\mathbf{P} \mathrm{o}\right.$ IMPRS-P) en final de palabra, no puede determinarse sin más datos a cuál de los tres morfemas mencionados corresponde $-a$. A estos debe sumarse $-j i$ 'dentro de' en el interior de frase fonológica (no ante pausa, donde se distingue de los demás por la inserción de [?] final).

Entre una base terminada en vocal y una P comenzada en vocal se inserta $y$, lo cual a su vez provoca desplazamientos en la altura de las vocales. Para este y otros procesos fonológicos, véase el APÉNDICE.

Las PP -'e, -ji y kisyé'e rechazan los complementos humanos, por lo cual no tienen una forma pronominal para los participantes del acto de habla $(\mathrm{PAH}) .^{5}$

En la TABLA 2 se exponen los pronombres personales que son diferentes de los posesivos, aglutinados o amalgamados con la $\mathrm{P}$ correspondiente.

${ }^{4}$ De hecho $-e j$ y $-e$ son isomórficos en la mayoría de los contextos, pero sólo el segundo puede producir palatalización de la consonante siguiente, p. ej. en el morfema de pasado remoto /peh/: /-eh-peh/ $=-e-p e_{5}$ vs. /-ey-peh $\rightarrow-$ ey-p ${ }^{y}$ eh/= $\left.-e-p i\right)$.

Sin embargo, podría haber excepciones: $i$-si'me' (/i-k em-Re/ 3S-agarrar+Loc.P) 'lo agarra' admite un participante humano como complemento. Cuando éste es un PAH adopta las formas pronominales correspondientes al locativo general -ham: $\boldsymbol{i}$-sim-k'i'm (/i-k ${ }^{\mathrm{y}} \mathbf{e m}-\mathbf{k}^{\mathrm{y}}$ 'em/ 3S-agarrar-1sg+P) 'me agarra'. 
TABLA 2: $\mathrm{P}$ y pronombres oblicuos $\neq$ posesivos

\begin{tabular}{|c|c|c|c|c|}
\hline & $1 \mathrm{sg}$ & $2 \mathrm{sg}$ & $1 \mathrm{pl}$ & $2 \mathrm{pl}$ \\
\hline $\boldsymbol{e} \boldsymbol{e j} /-\mathrm{eh} / \mathrm{INST}$ & $-\boldsymbol{k}^{\prime} \boldsymbol{i} / \mathrm{-k}^{\mathrm{y}}{ }^{\prime} \mathrm{eh} /$ & -'aj/-(?) $\alpha \mathrm{h} /$ & -ts'ej /-ts'eh/ & 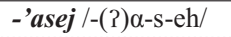 \\
\hline$-e /-e^{h} /$ DIST & $-\boldsymbol{k}^{\prime} \boldsymbol{i} / \mathrm{k}^{\prime}$ ey $/$ & -'a/-(?) $\alpha y^{\mathrm{h}} /$ & -ts'e/-ts'ey / & 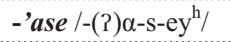 \\
\hline $\begin{array}{l}\text {-jam } \\
\text { /-ham/LOC.G } \\
\text {-a/-ah/ OR }\end{array}$ & $\begin{array}{l}\text {-k'im } \\
\text { /-k' }{ }^{\mathrm{y}}{ }^{\mathrm{em}} /\end{array}$ & -'am /-(?) $\alpha \mathrm{m} /$ & -ts'em /-ts'em/ & -'asem /-(?) $\alpha$-s-em/ \\
\hline
\end{tabular}

La segmentación de los pronombres no es del todo clara en algunos casos, por lo cual he preferido no segmentarlos y considerarlos amalgamados con $\mathrm{P}$, excepto por la forma de segunda persona del plural ' $a$-s- (2-PL). Las formas en $/ \mathrm{m} \#$ / glotalizan regularmente ante pausa, cf. 'wen-k'i'm 'dame' (véase APÉNDICE).

En las formas pronominales de la TABLA 2 se percibe generalmente un acento secundario y, en la pronunciación pausada de la elicitación, este acento puede tener la misma intensidad que el del verbo y aun puede percibirse una pausa entre ambos, p. ej. 'wen-k'i'm [ '?wen 'k'i?m ] 'dame'. Esto inclina a considerar que las formas de la TABLA 2 son palabras fonológicamente independientes que, al ser monosilábicas, tienden a apoyarse en la palabra precedente en el curso de la frase fonológica y debilitar su acento de palabra.

A diferencia de lo que se ha postulado para el maká (Gerzenstein 1994) y el nivaclé (Fabre 2010), no veo en chorote razones para segmentar un pronombre de tercera persona en las formas básicas (primera columna) de la TABLA 2 p. ej. /-e-y h/ como '3-DIST', ya que la forma /-ey/ se liga a nombres (cf. /'wet-ey/ $\rightarrow$ 'wet-e 'al/del lugar'), de modo que en tales casos no hay modo de justificar el análisis de /-e-/ como pronombre. Además, esta supuesta "forma pronominal" de algunas P (/-e-/ por caso) coocurre también con el pronombre de segunda persona del plural, cf. -'a-s-e (2-PL-DIST) 'a/de ustedes'. (De todos modos, según se verá en la sección 3, estas formas básicas como /-eyh/ podrían reflejar, en algunos casos, P más un complemento pronominal (nulo) subyacente).

La TABLA 3 muestra los pronombres personales que son idénticos a los posesivos, junto con el aplicativo/adposición correspondiente.

TABLA 3: $\mathrm{P}$ y pronombres oblicuos $=$ posesivos

\begin{tabular}{|c|c|c|c|c|c|c|}
\hline & & $1 \mathrm{sg}$ & $2 \mathrm{sg}$ & $3(=\mathrm{A})$ & $1 \mathrm{pl}$ & $2 \mathrm{pl}$ \\
\hline$-k^{\prime} i$ & DISTR, COM & $i-j y i-k^{\prime}{ }^{\prime}{ }^{\prime}$ & $a-j a ́-k^{\prime} i$ & $j l-e^{\prime}-k^{\prime} i{ }^{\prime}$ & $s-\dot{e}-k^{\prime} i{ }^{\prime}$ & $a s-e^{\prime}-k^{\prime} i$ \\
\hline$-j a p$ & junto.a & $i$-jyap & $a-j a p$ & jl-ajáp & s-ajáp & $a s-a p$ \\
\hline apée & sobre & $i$-ti-pe'e & $a$-té-pe'e & ji-ti-pe'e & si-ti-pe'e & as-té-pe'e \\
\hline k'iyé & BEN & i-ts'yoye & $a-k$ 'yoye & ji-k'yoye & si-k'yoye & as-k'yoye \\
\hline ilyám & viniendo & $i$-jyí-lya'm & $a-j e ́-l y a ' m$ & $?$ & $s-\dot{e}-l y a ' m$ & as-é-lya'm \\
\hline kyajwẹ & bajo & $i$-syajwe & a-kyajwe & ji-kyajwe & si-kyajwe & as-kyajwe \\
\hline
\end{tabular}

("=A" en la columna de la tercera persona indica correferencialidad con A)

Las formas de la cuarta columna (“3(=A)") sólo ocurren cuando el complemento de $\mathrm{P}$ es correferencial con A (véase sección 5); en otro caso, el complemento de tercera persona es nulo y las formas superficiales son las de la primera columna ( $-k^{\prime} i,-j a p$, etc). 
Obsérvese que en muchos casos puede identificarse un morfema entre $\mathrm{P}$ y el pronombre, presumiblemente un nombre fosilizado que habría denotado una parte del cuerpo, p. ej. /-he/en las formas pronominales de - $k$ ' $i$, realizado -jyi en $i$-jyi-k' $i$ ' 'conmigo' y -e- en $j l-\hat{e}-k^{\prime} i$ ' 'consigo'. Posiblemente el mismo análisis valga para k'iyé (dialectal k'yoyée e), -k'yoye, que podría analizarse diacrónicamente como N-P: k'yo-ye (/k'yo-ah/) donde $k^{\prime} y o$ - sería un nombre fosilizado, cf. -k'yo-te 'oreja', -k'yo-lo' 'mejilla'. 6

\section{ORDEN DE PALABRAS}

Cuando $\mathrm{P}$ es polisilábico es una palabra fonológicamente independiente y, bajo ciertas condiciones, también morfológicamente. Un complemento precedido de determinante sigue a una P polisilábica (4); en adelante, llamaré "pesados" a tales complementos. En (5) el complemento carece de determinante (es "liviano") y en consecuencia precede a P; además, el complemento de tercera persona disjunto de A es nulo, (6). Según se verá inmediatamente, (4)-(6) representan estructuras sustancialmente diferentes, y no sólo en relación con el orden de palabras.

$$
\begin{aligned}
& Y-i \quad(\mathrm{ADV}) \text { apé'e (ADV) 'ni } \quad \text { ajnát. } \\
& \text { 3S-estar } \quad \text { sobre } \quad \mathrm{D}_{1} \quad \text { tierra } \\
& \text { 'Está sobre la tierra' (Es decir, 'existe') }
\end{aligned}
$$

$$
\begin{array}{ll}
Y-i \quad(\mathrm{ADV}) & \text { a'lénta (*ADv) } \\
\text { apé'e } \\
\text { 3S-estar caballo } & \text { sobre } \\
\text { 'Anda (lit. 'está') a caballo' } &
\end{array}
$$

$$
\begin{array}{lr}
Y-i \quad \text { ADv) } & \text { apé'e } \\
\text { 3S-estar } & \text { sobre } \\
\text { 'Está sobre él/ella/eso' }
\end{array}
$$

El hecho de que P es palabra morfológicamente independiente en (4) y (6) queda evidenciado por el material que puede intervenir entre él y el verbo o el SD complemento; ADv entre P y su complemento léxico puede ser pet 'a su vez, también', jiwẹ́k 'finalmente', wat 'tanto, con fuerza', etc., y entre $\mathrm{V}$ y $\mathrm{P}$ los ya mencionados más diversos morfemas TAM como $p e$ 'pasado remoto', etc. Entonces, $\mathrm{P}$ no es aquí una preposición canónica, en tanto no es necesariamente adyacente a su (supuesto) complemento. Esto conduce a una observación importante: apée 'sobre' debe analizarse en (4) más bien como P más un complemento pronominal nulo doblado por el "complemento" léxico 'ni ajnát 'la tierra', que sería entonces en realidad un adjunto. ${ }^{7}$ En cualquier caso, por simplicidad, continuaré

${ }^{6}$ Las formas comenzadas en vocal toman una oclusión glotal inicial no fonológica, no representada aquí -a diferencia de TABLA 2- ya que se trata de palabras plenamente independientes y escritas separadamente; la realización de nam ijyik'i 'viene conmigo' es regularmente [na?mi'hyík'î], donde /m\#il $\rightarrow$ [?m].

${ }^{7}$ Este análisis es una adaptación de Jelinek (1984), retomado en Baker (1996), entre otros, donde se postula que, en algunas lenguas, los predicados (verbales) toman argumentos pronominales que son doblados por SSNN/SSDD adjuntos. 
denominando $\mathrm{P}$ a apée 'sobre' y complemento de $P$ a sintagmas como 'ni ajnát en construcciones como la de (4). En (5), en cambio, no puede intervenir material entre P y su complemento nominal, de lo que se infiere que $\mathrm{P}$ es allí una posposición. Si bien, al ser posposición, no constituye una palabra morfológica, es aún una palabra fonológica, ya que porta acento de intensidad propio, al igual que su complemento. Este complemento puede ser además fonológicamente nulo (6).

En cambio, si P es monosilábico se liga obligatoriamente, sea a su complemento ( $\mathrm{P}=$ posposición) o al verbo ( $\mathrm{P}=$ aplicativo). Las reglas que determinan el orden de $\mathrm{P}$ monosilábico son las siguientes:

(7) Orden sintáctico de P monosilábico

a. Si el complemento es un pronombre personal nulo, P se liga a él (pro+P). A su vez, (i)- si la palabra fonológica formada por pro+P es un monosílabo, es obligatoriamente adyacente al verbo, véase (8)

(ii)- si la palabra fonológica formada por pro+P es un polisílabo, es morfológicamente independiente, véase (9) (aunque véanse excepciones en (15).

b. Si hay un complemento léxico y éste es (i) "liviano” y además (ii) (típicamente) locativo, $\mathrm{P}$ se liga a él ocurriendo como posposición; véase (10).

c. En cualquier otro caso, P se liga al verbo; véanse (11)-(14) (aunque véase también las excepciones de (16) y (17))

Ejemplos:

(8) I-tyant'yej-k'i

3S-conocer-1sg+INST

'Me conoce'

(9) $\quad Y-i$

(ADv) i-jyap

3S-estar 1sg.POS-junto.a

'Vive (lit. 'está') conmigo'

(10) $\quad Y-i$

(ADv) in-kijmaye

'wet-'e

$(=(2))$

3S-estar POS.IND-agarrar+NMZ

'Está en el ingenio' (lit. 'lugar de trabajo')

[3Pos]lugar-LOC.P

(11) $\quad Y-i-' i$

(ADv) ja-na

in-lakijnyeye

$(=(1))$

3S-estar- LOC.P

$\mathrm{f}-\mathrm{D}_{2}$

POS.IND-jugar+NMZ

'Está en la fiesta'

(12) I-syet

$a j-a$

(ADv) ja-na pelóta

mi-pie

$\left[1 \mathrm{~S}_{\mathrm{A}}\right]$ golpear-INST

$\mathrm{f}-\mathrm{D}_{2}$ pelota

'Pateo (lit. 'golpeo con mi pie') la pelota' 
(13)

$Y-i-i$

3S-estar-LOC.P

'Está (en ese lugar)'

(14) I-tyant'yej-e

3S-conocer-INST

'Lo conoce'

En (10) el complemento es "liviano" "y locativo, por lo que P se liga a él, pero en (11), aunque locativo, es "pesado" y en consecuencia P se liga al verbo. En (12), aunque "liviano", no es locativo, por lo que P se liga también al verbo y el complemento "liviano" $i$-syet 'mi pie' ocurre a la izquierda de éste. En (13) y (14) los complementos (de tercera persona) son fonéticamente nulos, por lo que $\mathrm{P}$ se liga al verbo sin importar si el complemento es locativo o no.

La regla (7a) explica el orden de pro+P en términos de cantidad de sílabas; sin embargo, existe una excepción. Cuando pro es $2 \mathrm{pl}$ y $\mathrm{P}$ es una de las $\mathrm{P}$ monosilábicas que toman pronombres diferentes a los posesivos (cf. TABLA 2), entonces pro+P da como resultado un bisílabo que, sin embargo, es obligatoriamente adyacente al verbo (la forma subyacente de la secuencia subrayada -'wa-s-e aquí se muestra al final de la primera línea del ejemplo).

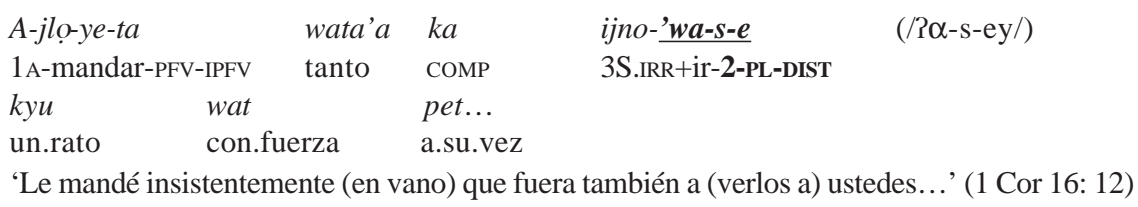

En (15) -’(w)a-s-e (2-PL-DIST) es obligatoriamente adyacente al verbo, según muestra el hecho de que los adverbios que le siguen no pueden interponerse. La explicación que propongo es la siguiente: en todas las demás personas, pro más una $\mathrm{P}$ de esta clase (aquéllas que no seleccionan posesivos) da como resultado un monosílabo (cf. - $k$ ' $i$ ' $1 \mathrm{sg}+\mathrm{INST}$ ', etc., cf. TABLA 2), por lo que la presión analógica fuerza a $2 \mathrm{pl}+\mathrm{P}$ a comportarse como el resto del paradigma. De aquí se obtiene otra conclusión: la cantidad de sílabas no es un criterio absoluto, sino que está subordinado a un criterio morfológico. En definitiva, el orden de pro+P no está determinado fonológicamente sino morfofonológicamente. Pese a que la explicación de los hechos mediante la regla (7a) más una excepción no es del todo económica, he preferido enunciar los hechos de este modo ya que la regla expresa una generalización importante: con la excepción señalada, P más su complemento se comporta igual que $\mathrm{P}$ en cuanto al orden de palabras, siendo la cantidad de sílabas $-\mathrm{y}$ no la presencia o ausencia de un complemento- lo que determina el orden.

\footnotetext{
${ }^{8}$ Inkijmaye (ji-)'wet'ingenio' lit. 'trabajo (su) lugar' puede considerarse una clase de compuesto o una construcción genitiva; sin embargo, es un complemento "liviano" ya que no está precedido de determinante.
} 
Una excepción a (7b-c) la constituye la construcción - aján N-ej 'ser.sabio N-INST', donde - ej 'INST' (aquí funcionando como tema) ocurre como posposición pese a que su complemento no es locativo (16). El ejemplo (17) es una excepción en sentido contrario: P ocurre como aplicativo pese a que su complemento es locativo y "liviano".

a.

$\begin{array}{ll}\text { 'Yijén } & \text { jl-ak-e } \\ \text { 3S+ser.sabio } & \text { 3POS-comida-INST }\end{array}$

'Sabe rebuscárselas (una persona)'; 'ya sabe comer solo (un niño o animal pequeño)', lit. 'conoce su comida'

\section{b. 'Yijén jl-osik-i \\ 3S+ser.sabio 3POS-alma-INST}

'Es sensato/considerado', lit. 'conoce su alma'

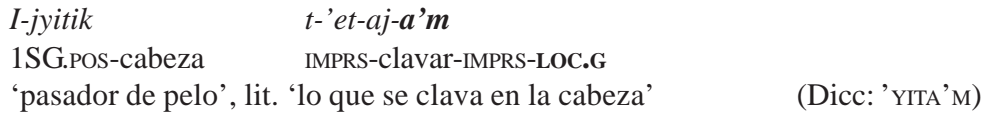

Téngase presente que, en el caso más frecuente, - ej ocurre ligado a -aján 'ser sabio', cf. 'yijén-e 'sabe', seguido por un complemento "pesado" o nulo.

En relación con la naturaleza sintáctica de los complementos "pesados" y "livianos" de P, una hipótesis es suponer que los primeros son sintagmas (SX) y los segundos núcleos sintácticos $\left(\mathrm{X}^{\circ}\right)$, incluyendo aquí compuestos o construcciones posesivas (véase n. 8). De este modo, los casos en que $\mathrm{P}$ ocurre como posposición podrían explicarse por adjunción de núcleo a núcleo (Baker 1988), es decir, incorporación a P de su complemento (18), mientras que cuando el complemento es un SX existen en principio dos explicaciones posibles. Según una hipótesis, la estructura subyacente podría ser similar a la de un sintagma adposicional normal, con la salvedad de que $\mathrm{P}$, al no poder ligarse a un SX, debe ligarse al verbo, quizá postsintácticamente, ${ }^{9}$ véase (19a). Según otra hipótesis, la estructura podría ser como la de (19b), donde lo que superficialmente denomino "aplicativo" sería en realidad P más un pronombre fonológicamente nulo y, siendo pro+P un monosílabo, debe ligarse al verbo, quizá también postsintácticamente; en este caso, el "complemento léxico" jana inlakijnyeye 'la fiesta' sería un adjunto. Es decir, (19b) representa una estructura similar a la propuesta más arriba para (4).

\footnotetext{
9 La derivación sintáctica se bifurca en un punto: una "rama" continúa la derivación hacia la forma lógica (FL) y otra hacia la forma fonética (FF). Los movimientos postsintácticos, admitidos en corrientes como la Morfología Distribuida (Halle y Marantz 1993 y trabajo subsiguiente), tienen lugar en esta última y no afectan, en consecuencia, la interpretación semántica.
} 
(18)

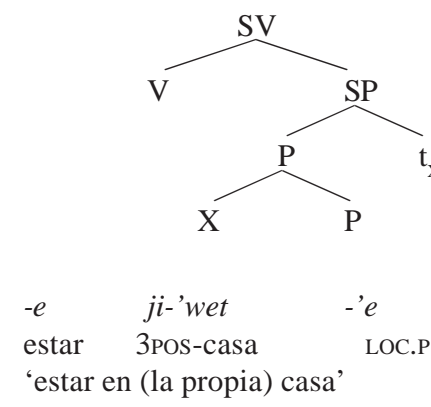

(19) a.

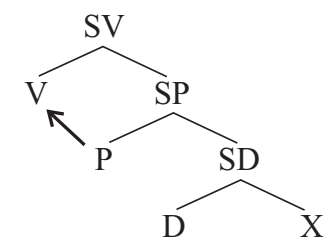

b.

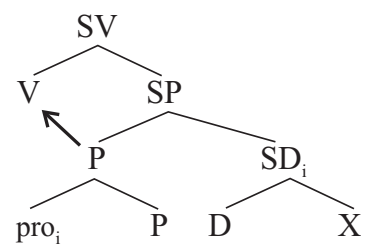

'Í $d$ ' (cf. (1)=(11))

Los datos favorecen en parte la hipótesis de que los complementos "pesados" son SX y los "livianos" son $\mathrm{X}^{\mathrm{o}}$, aunque aún debe explicarse la estructura de oraciones como (12), donde el complemento $X^{\circ}$ de $P$ no se incorpora a $P$ (véase n. 11). Utilizo deliberadamente la notación "X" en (18)-(19), dejando abierta la cuestión acerca de la clase de palabra a la que pertenece. Ji'wet 'su casa' en (18) se comporta del mismo modo que un $\mathrm{N}$ como tewuk 'río' pese a que la presencia del posesivo sugiere un SD.

Por otra parte, algunas PP ligadas a un complemento dan lugar a lexicalizaciones. Así, el instrumental o tema - ej ligado a los pronombres demostrativos 'naka 'éste (ostensible)', paka 'aquél (inaccesible)', jaka 'ese (conocido no visible)' y al pronombre interrogativo tepa 'cómo/dónde' forma las construcciones lexicalizadas con valor temporal 'naka-yi 'actualmente/entonces', ${ }^{10}$ paka-yi, jaka-yi 'en aòquel tiempo' y tepa-yi 'cuándo', respectivamente. A su vez, el locativo -ji ligado a noò'oÚñt 'tarde' da noò'oÚñt-i' 'ayer', etc. Cuando esto sucede, el lexema puede ocurrir al comienzo de la cláusula (20), (22) o incluso al final (21).

$$
\begin{aligned}
& \text { 'Na-ka-yi je i-stye ni sijwónye kajéyepa... } \\
& \mathrm{D}_{1}-K A \text {-INST NEG 3S-ser.difícil } \mathrm{D}_{2} \quad \text { sal } \quad \text { porque... } \\
& \text { 'Actualmente no escasea la sal, porque...' } \quad \text { (Sake' 18) }
\end{aligned}
$$

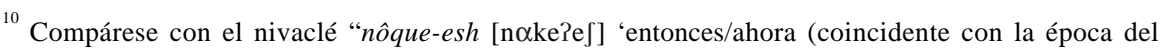
relato)", (Fabre 2010). 


$\begin{array}{ll}\mathrm{Ti} & p a j-k ' i \\ \text { COMP } & {[3 \mathrm{~S}] \text { lleva.tiempo-DISTR }} \\ \text { Je } & i \text {-yo-'até } \\ \text { NEG } & \text { 3S- } v \text {-así }\end{array}$

$i$-stye

3S-ser.difícil

'na-ka-yi.

$\mathrm{D}_{1}-$ KA-INST

'Antes era dificil conseguir sal. No era como ahora'
¿Tepa-t'i-yi ja-pá-k
jloma
ti
kya-k
ja-wo?
Qu-CONJ-INST $\mathrm{f}^{-\mathrm{D}_{6}-K}$
día
COMP $\mathrm{D}_{4}-K$
¿Cuándo será el día en que sucederá esto?
PRSP- $3 S+v$
(Mt 24:3)

ni sijwónye.

$\mathrm{D}_{2}$ sal

Un hecho algo desconcertante, sin embargo, es que puede intervenir morfología de TAM entre P y los pronombres mencionados arriba. En (22) el clítico conjetural t'i se interpone entre el complemento y $\mathrm{P}$, aunque esto puede explicarse en tanto se trata de un clítico de segunda posición, de modo que probablemente no interrumpe el constituyente sino que adopta su posición postsintácticamente. En cambio, más difícil es explicar el morfema de IRREALIS propio de predicados nominales entre el pronombre

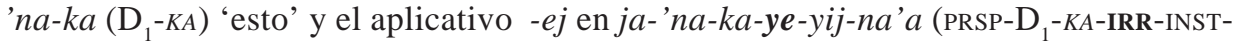
FPROX) 'para que (FUTURO)'.

También se documentan sintagmas posposicionales desplazados a la izquierda fuera de los casos lexicalizados mencionados: véase (23), donde el SP aparece topicalizado.

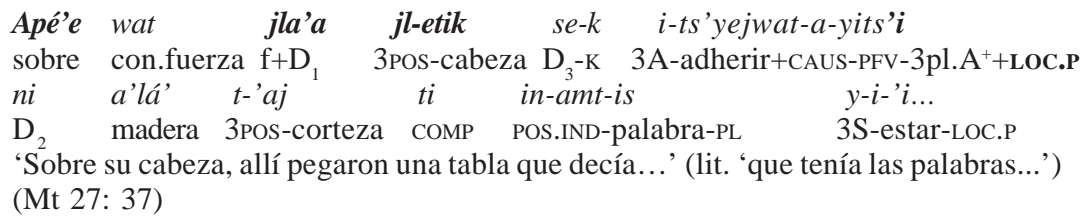

En cuanto al orden del complemento léxico de $\mathrm{P}$ aplicativo respecto de $\mathrm{O}$ léxico, el primero aparece más cerca de la palabra fonológica conformada por $\mathrm{V}$ y $\mathrm{P}$ en (24), aunque en (25) sucede lo contrario. Es posible que el rol de cada argumento incida en esto, aunque es necesaria mayor investigación.

$$
\begin{aligned}
& \text { I-jyos-am ni-wa ji-kapis-ki' ni-wa ji-sijn-a si'yús } \\
& \text { 3A-cargar-LOC.G } \mathrm{D}_{2} \text {-PL.nh 3Pos-troja-f } \quad \mathrm{D}_{2} \text {-PL.nh 3Pos-asar+NMZ-PL pescado } \\
& \text { 'Guardaban en las trojas sus asaduras de pescado.' } \quad \text { (Sake' 26) }
\end{aligned}
$$

(25) I-'yen-kin-e na-pọ kya'li-s nyu-wa im-pel-is

3A-mirar-CAUS-INST $\quad \mathrm{D}_{2}$-PL.h niño-PL $\quad \mathrm{D}_{2}$-PL.nh $\quad$ POS.IND-sombra-PL(=película)

'Les hizo mirar una película a los chicos'

En (25) napo kya'lis 'los chicos' es O, cf. si-'yen-kin-e... 'me hizo mirar'. Cuando P es adposición el SP suele ocurrir más cercano al verbo que $\mathrm{O}$, aunque también he documentado el orden inverso: 
Carol: Aplicativos/Adposiciones en Chorote (MATAguayo): AlgunOS...

$\begin{array}{llll}\text { a. A-jo-jwat } & \text { La.Paz-e } & n a & \text { Juan } \\ \text { 1A-ir-CAus } & \text { La.Paz-DIST } & \mathrm{D}_{2} & \text { Juan } \\ \text { 'Mandé a Misión La Paz a Juan' } & \\ \text { b. A-jo-jwat } & n a & \text { Juan } & \text { La.Paz-e } \\ \text { 1A-ir-CAus } & \mathrm{D}_{2} & \text { Juan } & \text { La.Paz-DIST } \\ \text { 'Í } d \text { ' } & & & \end{array}$

Por último, vale la pena volver sobre los instrumentales y algunos otros argumentos que en chorote siempre aparecen introducidos por $\mathrm{P}$ aplicativo y nunca adposicional. En efecto, los aplicativos instrumentales y algunos otros poseen ciertas características sintácticas que los distinguen de los demás y que, en cierto modo, los acercan a los causativos. El argumento que introducen es frecuentemente "liviano" y, al no ser locativo, aparece normalmente en posición preverbal y no entre el verbo y P (cf. 7b-c):
a.
I-syet
1sg.POS-pie
$a j$
ja-na
pelóta
'Pateé la pelota' (lit. 'Golpeé la pelota con mi pie')
b. $* A$
i-syet-e
ja-na
pelóta
[1A]golpear
1sg.POS-pie-INST
$\mathrm{f}-\mathrm{D}_{2}$
pelota

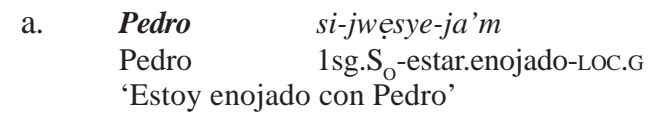
b. *Si-jwẹsye Pedro-ja'm
1sg.S $\mathrm{O}_{\mathrm{O}}$-estar.enojado Pedro-LOC.G

(28)

Más aún, esta clase de "argumento aplicado" puede incluso ocurrir delante del sujeto de un verbo inacusativo:
At'és
i’nyó
i-jyum-e
bebida.alcohólica persona
3S-estar.borracho-INST

'El alcohol emborracha al hombre', lit. ‘el hombre se emborracha con alcohol' (500: AT'ES)

Pylkkänen (2002) señala una característica común a los aplicativos instrumentales, benefactivos, malefactivos y similares, por un lado, y a los causativos por otro: ambos introducen un argumento que se vincula con todo el sintagma verbal, a diferencia de otros aplicativos (destinatario [ $\mathrm{goal}$ ], fuente [source], etc.) que relacionan el nuevo argumento sólo con el objeto directo. Así, en Juan le hizo el informe a Luis -suponiendo que el clítico doblado le sea un tipo de aplicativo- una interpretación de a Luis como destinatario implica simplemente que Luis es quien debe recibir el informe (por ejemplo, si Juan es un empleado a quien su jefe Luis se lo había pedido); en cambio, una lectura benefactiva de a Luis implica que Luis es beneficiario del evento de hacer el trabajo, aunque no sea necesariamente el destinatario del informe (por ejemplo, si ambos son compañeros de trabajo y Juan, como favor, realizó una tarea -redactar un informe- que debía hacer Luis). En razón de su diferente posición en la estructura sintáctica -y, consecuentemente, de su 
diferente alcance-Pylkkänen llama "aplicativos altos" a los de este último tipo, ya que se ensamblarían por encima de la raíz verbal ${ }^{11}$ al igual que los causativos, y "bajos" a los del primero, que se ensamblarían debajo de la raíz verbal. Esta hipótesis luce promisoria para explicar el comportamiento sintáctico particular de los complementos de P en (27)-(29), si bien muchos detalles requieren mayor explicación. ${ }^{12}$

Si la explicación de la morfosintaxis de (27)-(29) mediante la hipótesis de los "aplicativos altos" está bien encaminada, lo que determinaría que $\mathrm{P}$ se ligue al verbo y no al nombre cuando el complemento es "liviano", como en (27)-(29), no sería el carácter no locativo de éste (cf. 7b) sino su carácter de argumento aplicado "alto". Sin embargo, falta comprender mejor qué sucede con los argumentos "livianos" de aplicativos "bajos" no locativos; (16) es uno de tales casos, aunque son necesarios muchos más ejemplos para arribar a conclusiones firmes. Otro caso afín lo constituyen los dativos destinatario y fuente de verbos como 'dar' y 'quitar', respectivamente, pero no documento dichos argumentos que ocurran como sintagmas "livianos"; en tales predicados, si uno de los argumentos es liviano, éste es O, no el destinatario, p. ej. si'yús awejna'm na Juan 'le doy pescado a Juan'.

${ }^{11}$ En relación con la hipótesis de que los complementos "livianos" de P son $\mathrm{X}^{\mathrm{o}}$, la hipótesis de los aplicativos "altos" permitiría explicar por qué aquéllos no se incorporan a $\mathrm{P}$ cuando $\mathrm{P}$ corresponde a un aplicativo "alto". La razón es que entonces tendría lugar una adjunción de núcleo a núcleo de $\mathrm{V}$ a $\mathrm{P}$ "alto", de modo que desaparecería el contexto para la adjunción de N a P:

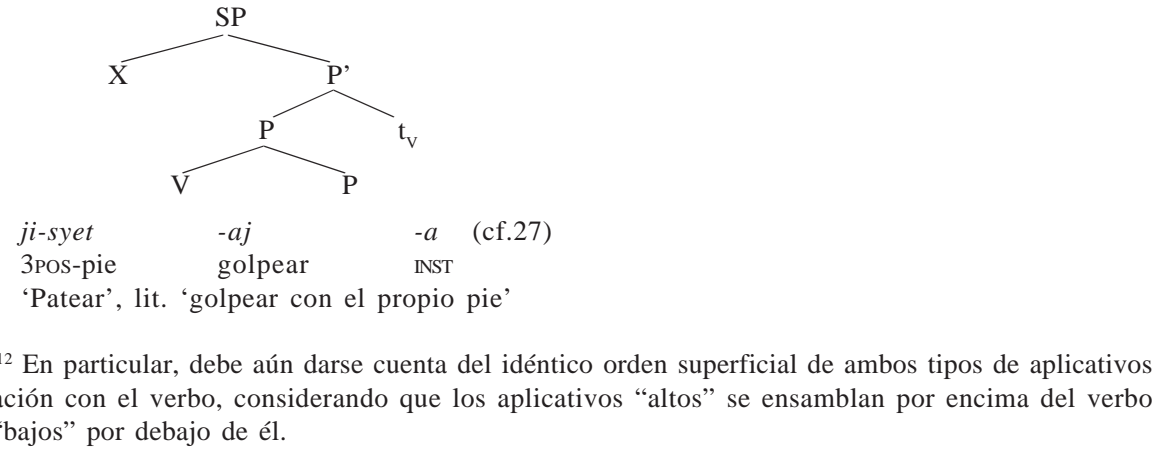
y los "bajos" por debajo de él. 
Carol: Aplicativos/Adposiciones en Chorote (Mataguayo): AlgunOS...

\subsection{Orden de los aplicativos}

Las PP tienen un orden relativo rígido cuando funcionan como aplicativos; éste se expone en TABLA 4.

TABLA 4: Orden de los aplicativos

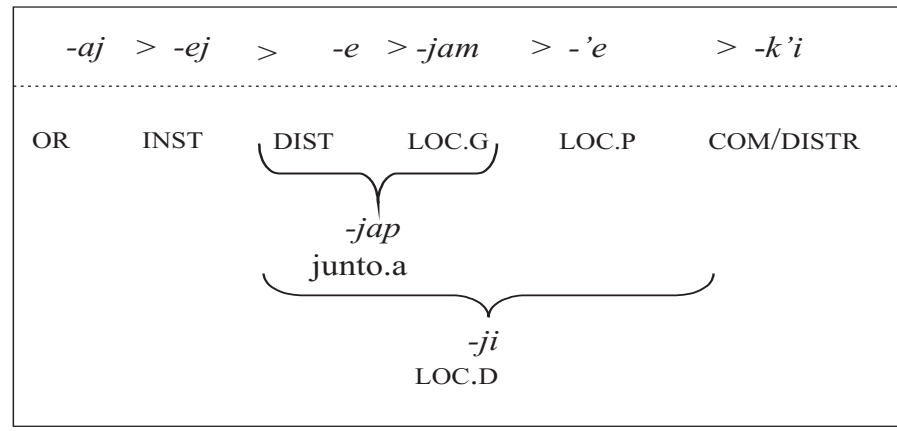

A continuación se ofrecen ejemplos que ilustran el orden; recuérdese que la forma superficial de $-e$ es /-eyh/, lo cual explica la palatalización de la consonante siguiente.

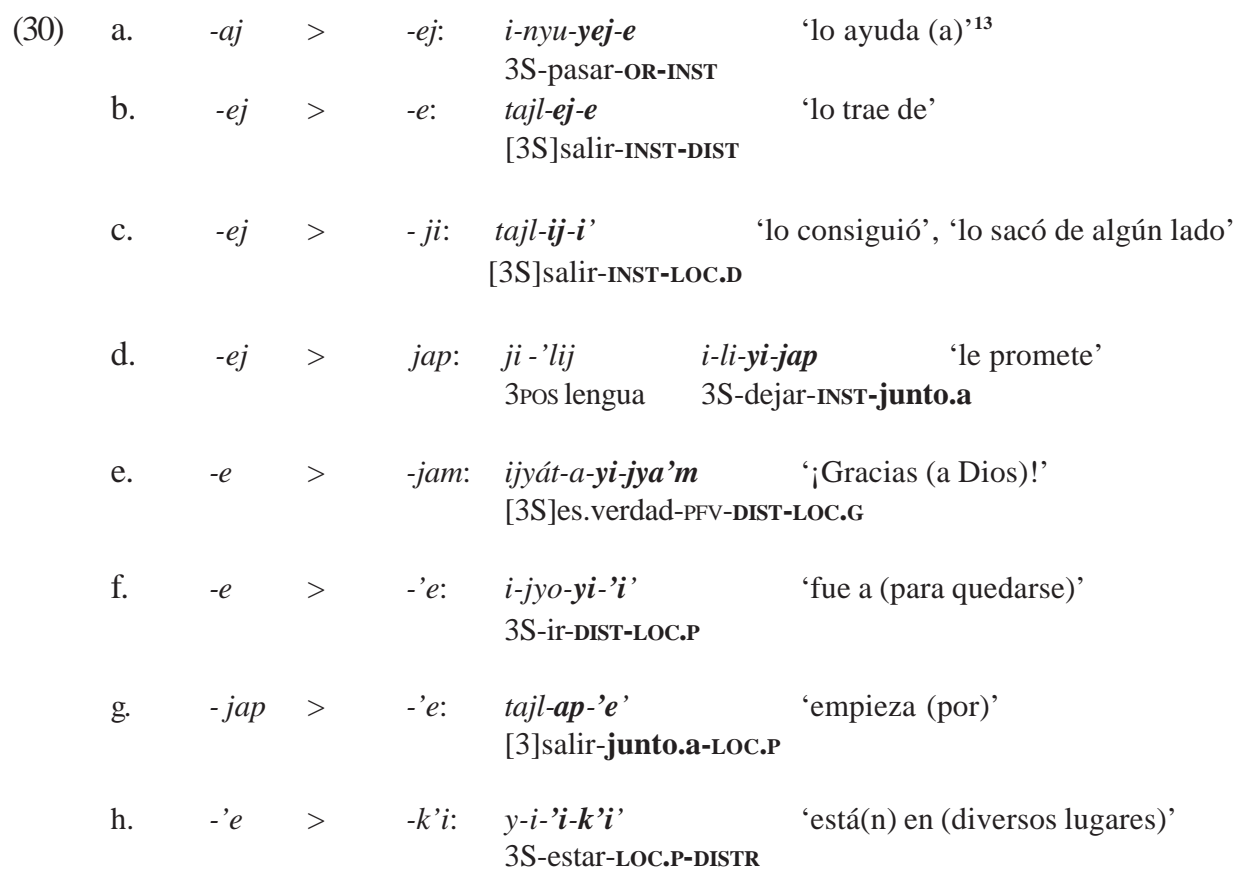

${ }^{13}$ En la variedad iyo' (a)wujwa' la forma correspondiente es $\boldsymbol{i}$-nyu-waj-a. En esta variedad se inserta $w$ entre vocales en lugar de $y \mathrm{y}$, en consecuencia, no tiene lugar el desplazamiento de altura vocálica que ocurre en iyojwa'aja'. 
Este orden sólo vale cuando los aplicativos toman un complemento de tercera persona. En cambio, si un aplicativo toma un PAH como complemento, aparece a la derecha del resto independientemente de cuál sea.

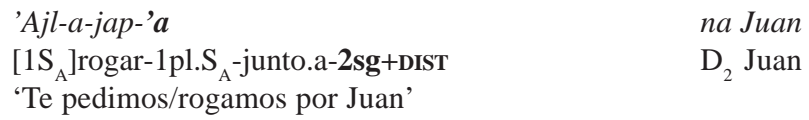

na Juan

$\mathrm{D}_{2}$ Juan

En (31) el distal $e(/$ ey $/)$, que normalmente precede a -jap, está amalgamado con el pronombre de segunda persona del singular (-'a) y en consecuencia aparece al final de la secuencia de aplicativos.

\section{CARACTERIZACIÓNGENERAL DE LOS COMPLEMENTOS DEP}

Los complementos léxicos de $\mathrm{P}$ que suelen ocurrir sin determinante (es decir, "livianos") son nombres como 'río', 'agua', 'tierra' (como 'el piso' o 'el mundo'), 'lugar/casa' (precedido de posesivo), partes del cuerpo, etc., es decir, entidades "familiares" a los participantes del acto de habla y discursivamente accesibles, hayan sido introducidos previamente en el discurso o no; obsérvese que estas propiedades son compartidas también por los pronombres personales, a los cuales $\mathrm{P}$ también se liga obligatoriamente.

Los nombres que refieren a personas también pueden ocurrir como complementos "livianos" de P; en mi material, cuando ello sucede P se liga al verbo y su complemento aparece a la izquierda de éste, véase (28). Cuando $\mathrm{P}$ ocurre ligado a un nombre que denota humanos, éste designa en general un lugar más bien que un participante humano, véase p. ej. (32)

$$
\begin{aligned}
& \text { Inti a-'we'en, a-wen kiláyi-yi } \\
& \text { quizá? 1A-ver 1A-dar criollo-DIST } \\
& \text { 'Si acaso encuentro [miel], la vendo (p. ej. en un almacén)' (Sake' 38) }
\end{aligned}
$$

En (32) kiláyi ‘criollo’ designa en realidad un lugar de criollos, p. ej. un almacén. Por otro lado, compárense (33a-b), donde el posesivo de tercera persona correferencial con el sujeto ocurre en un sintagma "liviano", mientras que el disjunto lo hace en uno "pesado".

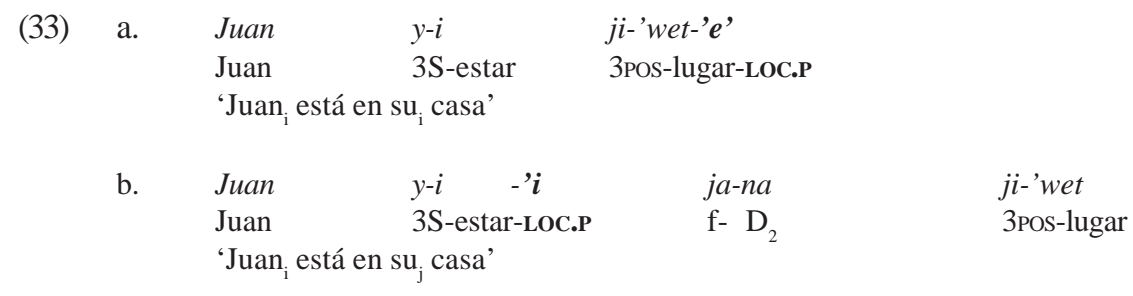

El complemento "liviano" de P también puede ser un pronombre demostrativo. En tales casos, P puede ligarse a él (34) o no (35): 
Carol: Aplicativos/AdPosiciones en Chorote (MATAguayo): AlgunOS...

(34)

$\begin{array}{ll}\text { I-tyet-e } & \boldsymbol{s e} \text { - } \boldsymbol{k} \boldsymbol{a} \text {-' } \boldsymbol{a} \text { ' } \\ \text { 3S-tirar-DIST } & \mathrm{D}_{3} \text {-KA-LOC.P }\end{array}$

'Lo tiró allá'

\begin{tabular}{|c|c|c|c|c|}
\hline $\begin{array}{l}\text { Inti } \\
\text { quizá } \\
\text { 'Ni-wá-k }\end{array}$ & $\begin{array}{l}i \text {-'wi'in } \\
3 \mathrm{~A}-\mathrm{ver} \\
j i \text {-tye }(j)\end{array}$ & $\begin{array}{l}p a \\
\mathrm{D}_{6} \\
i \text {-yom-a... }\end{array}$ & $\begin{array}{l}\text { k'ijlyó', } \\
\text { quirquincho }\end{array}$ & $\begin{array}{l}\text { kasọts’i } \\
\text { mulita }\end{array}$ \\
\hline $\mathrm{D}_{1}-\mathrm{pl} \cdot \mathrm{h}-K$ & 3POs-vista & 3S-planta & & \\
\hline
\end{tabular}

'Quizá encuentra un quirquincho o una mulita. En ésto(s) deposita su confianza/esperanza.'

(Lit 'fija su vista')

(Sake' 28)

En (34), a diferencia de (35), el complemento es claramente locativo y, esperablemente, se liga a $P$.

\section{STATUS FORMAL DE P Y SU COMPLEMENTO}

Postulo que, cuando P se liga al verbo, no hay incorporación sino cliticización superficial. Me baso para ello en el orden de los morfemas -P es muy periférico en la palabra para ser una adposición incorporada- $y$, además, en que su complemento no manifiesta las propiedades de un argumento aplicado canónico. En relación con lo primero, cuando $\mathrm{P}$ ocurre ligado al verbo sigue a los sufijos de TAM que cierran la palabra morfológica $-\mathrm{y}$ por ende a toda la morfología flexiva- o a clíticos TAM de segunda

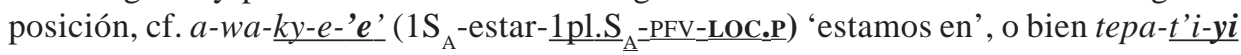
(QU-CONJ-INST) 'cuándo' en (22). Si se tratara de incorporación, se esperaría una posición contigua al tema verbal, en la medida en que la incorporación se concibe como una forma de composición.

En relación con lo segundo, el complemento de P no puede referirse mediante los proclíticos que marcan el objeto directo. El chorote posee un conjunto de prefijos pronominales o de concordancia verbal para el sujeto (A) y otro para el objeto (O) de un verbo transitivo, los cuales están sujetos a una jerarquía de persona $1>2>$ impersonal $>3$, de modo que sólo uno de ellos ( $\mathrm{A} \mathrm{u} \mathrm{O}$ ) aparece a la vez. Los prefijos y la jerarquía se ilustran abajo; sólo se indican los alomorfos ante consonante y sólo el singular.

\begin{tabular}{l|l|l|}
\multicolumn{4}{c}{} & \multicolumn{2}{l|}{ Tabla 5: prefijos verbales A y O } \\
\cline { 2 - 3 } \multicolumn{1}{c|}{} & A & O \\
\hline 1 & $a-$ & $s i-$ \\
\hline 2 & $j i-$ & $i n-$ \\
\hline IMPRS & $t i-\ldots-a j$ & -- \\
\hline 3 & \multicolumn{2}{|c|}{$i-{ }^{14}$} \\
\hline
\end{tabular}

${ }^{14}$ Dado que este prefijo sólo aparece cuando tanto el sujeto y el objeto son tercera persona, no puede establecerse en principio si marca el sujeto, el objeto o ambos. Cuando $i$ - ocurre con intransitivos lo hace tanto con verbos que marcan los PAH igual que A como con verbos que las marcan igual que $\mathrm{O}$. 
Tabla 6: jerarquía de persona en prefijos verbales; tema 'yen 'mirar'

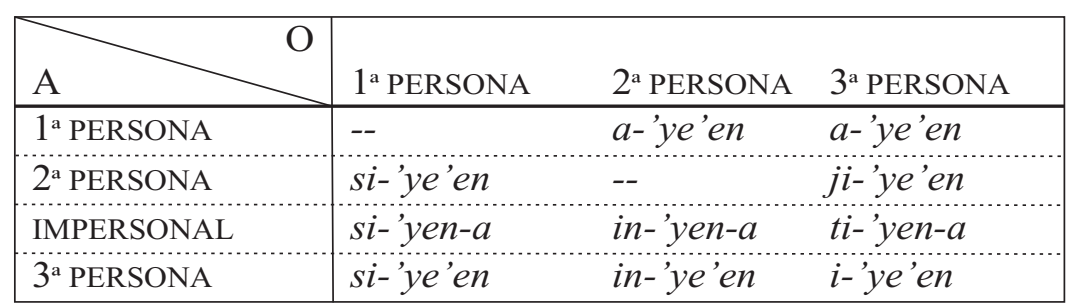

Ahora bien, retornando al complemento pronominal de $\mathrm{P}$, mientras que éste carece de expresión fonética en tercera persona (36a), en los PAH su pronominalización se realiza mediante un conjunto especial de marcas oblicuas (cf. 37a, TABLA 2 y TABLA 3) que difiere de las marcas de $\mathrm{O}$ (37b). Nótese que, en consecuencia, el complemento pronominal de P nunca interviene en la jerarquía de persona. Además, dicho complemento no habilita la ocurrencia del prefijo de $3 \mathrm{~A} i$-, $y$ - propio de los verbos transitivos (36b).

a.

t-an-e
3S-llamar-DIST
'Lo/la llama' b. *y-an-e

3A-llamar-DIST

b. *s-an-e

1O-llamar-DIST 3S-llamar-1sg+DIST

'Me llama'

En síntesis, el complemento de $\mathrm{P}$ es un argumento oblicuo y no un objeto directo.

Con todo, el complemento de $\mathrm{P}$ comparte algunas características con O: habilita la marca -is de tercera persona del plural y puede ser referido por el reflexivo/recíproco -ni(n) (39c). El sufijo -is marca el plural de la tercera persona A, pero también del sujeto de verbo intransitivo (S) cuando existe un segundo argumento introducido por P (38a); en cambio, cuando no existe tal segundo argumento, el plural de $\mathrm{S}$ se indica mediante otros morfemas (-jen en 38b).

a. 'yején-is- $i$

3S+ser.sabio-3pl.A $\mathbf{A}^{+}$-INST

'Lo saben'
i-'yen-a
3S-mirar-OR
'Lo cuida'
b. 'yejejne'n
$3 \mathrm{~S}+$ ser.sabio+JEN
'Son sabios'
b. $\quad i$-'yen-k'i'm
3S-mirar-1sg+oR
'Me cuida'
c. i-ni-'yen-a
3A-REFL-mirar-OR
'Se cuida'

Además, el complemento de $\mathrm{P}$ siempre puede relativizarse, al igual que un argumento central: 
CArol: Aplicativos/Adposiciones en chorote (Mataguayo): algunos...

$$
\begin{aligned}
& \begin{array}{lllll}
\ldots y-i-y i t s ' i & \text { syu-pa ajnát } \quad[\emptyset & \text { laj } & \text { i’nyó-ye-'e }]
\end{array} \\
& \text { 3S-estar-3pl.A+ }+ \text { LOC.P FOC-D } 6 \text { tierra [REL [3S]no.haber persona-IRR-LOC.P] } \\
& \text { 'Vivían en la tierra en la que no hay gente' (es decir, el desierto) (Hch 7: 44) }
\end{aligned}
$$

Por otro lado, la distinción entre complementos adposicionales y argumentos aplicados no parece corresponderse en chorote con una distinción entre adjuntos y argumentos. Un constituyente prima facie argumental como, por ejemplo, el complemento locativo de un verbo de movimiento, puede realizarse como complemento adposicional $((2)=(10))$, mientras que cualquier SN/SD puede realizarse como complemento de aplicativo (o adjunto que duplica un complemento pronominal) en tanto sea "pesado". En definitiva, no es claro que el "argumento aplicado" tenga necesariamente una relación más estrecha con el verbo que un complemento adposicional, lo cual también desfavorece la hipótesis de la incorporación. Más aún, si es correcto que los aplicativos que toman un complemento de tercera persona (como en (1)) son en realidad pro+P y que su "complemento" es en realidad un adjunto que dobla a pro, entonces los "argumentos aplicados" no serían en realidad argumentos sino adjuntos. De todas maneras, es posible que los argumentos aplicados instrumentales y algunos otros posean un status diferente al de los demás complementos de $\mathrm{P}$, según se expuso en $\S 3$.

Por último, dos observaciones. En primer lugar, el complemento pronominal de tercera persona es usualmente nulo, según se ha expresado más arriba, véase k'iyé en (41b). Sin embargo, las adposiciones/aplicativos que toman complementos isomórficos con los posesivos toman un complemento de tercera persona que es idéntico al posesivo de tercera persona cuando el complemento que refieren es correferencial con A (cf. TABLA 3), véase (41a).

a. ...i-jlyu-ye-t'i-'nij-pe
3A-mandar-PFV-CONJ-ITER-PREM
'(X) los mandó delante de sí', ' $\left(\mathrm{X}_{\mathrm{i}}\right)$ los mandó delante de sí ${ }_{\mathrm{i}}$ '

\begin{tabular}{lll}
$t i$ & i-nyajnat-en & ji- $\boldsymbol{k}$ 'yoye \\
COMP & 3A-ir.primero+CAUS-JEN & 3POS-BEN \\
& \multicolumn{2}{c}{ (Lc 10:1) }
\end{tabular}

k'iyé BEN ' $\left(\mathrm{X}_{\mathrm{i}}\right)$ los mandó delante de él.

En segundo lugar, P puede también ser complemento (o adjunto) de un nombre; éste

\begin{tabular}{|c|c|c|}
\hline$J a-w u j w-a$ & $t i$ & ja-jnots'ijni-ts' $i$ \\
\hline PRSP-3S+ser.grande-IRR & COMP & PRSP-estar.asustado+LOC.D-DISTR \\
\hline na-pọ i’nyó & apé'e 'ni & ajnát \\
\hline $\mathrm{D}_{2}$-PL.h persona & sobre $\quad D_{1}$ & tierra. \\
\hline
\end{tabular}
puede asumir una función predicativa (43) aunque no necesariamente.

\section{Ji-kat-k'i'm}

3Pos-contagio?-1sg+P

'Me contagia' (Lit. 'su contagio hacia mí')

(Dicc: 'YAKAT'E) 


\section{CUANDO P NO INCREMENTA LA VALENCIA}

Existen dos tipos de casos en los que P no incrementa la valencia o adicidad, es decir, no añade un argumento más a la cláusula. ${ }^{15}$ Por un lado están los casos como los de (44)-(47), donde $\mathrm{P}$ no introduce ningún argumento, o al menos no uno explícito.

a.

a.

i-mya-'a'
3S-dormir-LOc.P
'duerme'

$$
\begin{array}{ll}
\text { Tajl-i } i a-n a & \text { in-wom-ki' } \\
{[3 S] \text { salir-LOC.D } \mathrm{f}^{-D_{2}}} & \text { POS.IND-plantar-f } \\
\text { 'Germinó la planta' } &
\end{array}
$$
b. $\quad i-m y a-k^{\prime} \boldsymbol{i}-j i{ }^{\prime} n$
3S-dormir-DISTR-JEN 'duermen'

$$
\begin{aligned}
& \text { Ji-kyo } \quad \begin{array}{l}
\text {-jyey- } \boldsymbol{i} \\
\text { 3Pos-mano }
\end{array} \text { 3A-levantar-DIST } \\
& \text { 'Saluda de lejos' } \\
& \text { (Dicc: IJIE) }
\end{aligned}
$$

c. i-mya-('ni) 3S-dormir-ITER 'está(n) campeando', lit. 'duerme discontinuamente aquí y allá'

b. A-tajl-i na-pọ ijyá' 1A-salir-Loc.D $\quad \mathrm{D}_{2}$-PL.h otro 'Me separé del resto.'

En (44a) el locativo puntual -'e parece tener un valor meramente diacrítico, concretamente distinguir 'dormir' de 'campear', y sólo en el singular. En (45a) - ji 'dentro de' se utiliza, según Víctor Díaz (c.p), debido a que la planta brota del interior de una semilla; sin embargo la expresión no acepta un complemento explícito, por lo que considero que toma un complemento nocional prototípico fonéticamente nulo; obsérvese que la misma construcción sí es capaz de tomar un complemento explícito en otras expresiones, cf. (45b). En (46), el distal - $e$ ubica toda la eventualidad (levantar la mano) en una locación distante pero no introduce un argumento. Si se considera que $\mathrm{P}$ tiene las propiedades formales de una adposición salvo por el hecho de que debe ligarse al verbo, entonces el funcionamiento de $\mathrm{P}$ en estos casos no resulta tan inusual interlingüísticamente, ya que

\begin{tabular}{|c|c|c|c|c|c|c|}
\hline (47) & a. & $\begin{array}{l}i \text {-'yen-a } \\
\text { 3S-mirar-oR } \\
\text { 'lo cuida' }\end{array}$ & b. & $\begin{array}{l}i \text {-'yen-k'i'm } \\
\text { 3S-mirar-1sg+oR } \\
\text { 'me cuida' }\end{array}$ & c. & $\begin{array}{l}\text { *si-'yen-a } \\
\text { 1O-mirar-oR }\end{array}$ \\
\hline (48) & a. & $\begin{array}{l}i \text {-'ye'en } \\
\text { 3A-mirar } \\
\text { 'lo mira' }\end{array}$ & b. & $\begin{array}{l}\text { si-'ye'en } \\
\text { 1O-mirar } \\
\text { 'me mira/mirás' }\end{array}$ & & \\
\hline
\end{tabular}
se asemeja a ciertas preposiciones del inglés que no rigen un complemento, cf. on en put on your shoes 'ponete los zapatos' (cf. put them on the table 'ponelos sobre la mesa'), o a by en time goes by 'el tiempo pasa' (cf. I go by car 'voy en auto').

Por el otro lado, existen casos en los que $\mathrm{P}$ sí introduce un argumento pero, al mismo tiempo, "desaparece" el argumento $\mathrm{O}$ u otro argumento oblicuo.

\footnotetext{
${ }^{15}$ Entiendo aquí por "valencia" simplemente la cantidad de argumentos del predicado, considerando que $\mathrm{P}$ forma un predicado complejo con el verbo, mientras que por "transitividad" entiendo la propiedad de seleccionar un objeto directo marcado mediante prefijos $\mathrm{O}$.
} 
La raíz -’yen significa 'mirar' y es básicamente transitiva, según muestra (48). En (47), el aplicativo - $a j$ (superficialmente - $a$ en posición final) crea un predicado complejo traducible por 'cuidar', al tiempo que introduce un participante, el "cuidado": el complemento del predicado (la persona cuidada) está regido por - $a j$, según muestra (47b), y no por el verbo ya que, según muestra (47c), no puede correferirse mediante los prefijos $O$. En consecuencia, a la vez que $-a j$ ha introducido un argumento oblicuo, el verbo ha perdido su objeto directo.

En principio, existen dos interpretaciones posibles sobre la referencia del objeto "desaparecido":

(i) es correferencial con el argumento oblicuo explícito, aunque por algún motivo sólo este último puede expresarse superficialmente. Según esto, y considerando aquí - aj como una suerte de benefactivo, (47) podría parafrasearse por me mira para mí, donde sólo el oblicuo - $k$ 'i'm ('para mí') ocurre superficialmente;

(ii) es un objeto indefinido; (47) podría parafrasearse entonces por mira algo para mí. ${ }^{16}$ La hipótesis (i) es compatible con la hipótesis de $\mathrm{P}$ como originada en un verbo serial, de modo que el argumento compartido por ambos verbos (actualmente $\mathrm{V}$ y $\mathrm{P}$ ) sería marcado sólo en uno de ellos. A su vez, aún puede discutirse si el objeto "desaparecido" está presente en la sintaxis o no; al respecto, vale la pena notar que el verbo conserva la marca $i$ - $y$ - obligatoria para verbos transitivos (marca que, sin embargo, también ocurre en intransitivos). En cualquier caso, estas marcas son glosadas en el presente artículo como 3S y no como 3A cuando O "desaparece". Por otro lado, dentro de la hipótesis (i), podría considerarse que $\mathrm{P}$ "refina" el modo en que el participante es afectado o bien que $\mathrm{P}$ es una suerte de clasificador verbal. ${ }^{17} \mathrm{Si}$, otra vez, se considera que $\mathrm{P}$ tiene las propiedades de una adposición salvo por el hecho de que en ocasiones debe ligarse al verbo, entonces (48) tiene semejanza con ciertos usos de preposiciones del español, cf. mirar la pared y mirar a la pared, etc. La cuestión merece indudablemente mayor atención.

${ }^{16}$ Este análisis es similar al que puede proponerse en español para p. ej. Me comentó sobre el tema, es decir, Me comentó algo sobre el tema, en vista de la transitividad del verbo, cf. Me comentó sus intenciones.

${ }^{17}$ Fabre (2010) observa sobre un fenómeno similar en nivaclé: "De acuerdo con Aikhenvald (2000: 149-171) los clasificadores verbales relacionan argumentos nominales con sus índices verbales respectivos, sujeto o paciente, copiando en el verbo diversos rasgos semánticos del nombre (forma, consistencia, animacidad, etc). El nivaclé se aparta de este modelo en cuanto no es tanto la semántica del sujeto o del objeto que provoca la aparición de dichos morfemas, sino el cuadro espacial dentro del cual se desenvuelve el proceso, razón por la cual prefiero considerarlos como esencialmente locativos".

Estas consideraciones valen también para el chorote, si bien debería agregarse que no siempre $\mathrm{P}$ está relacionada con el "cuadro espacial". 


\section{CONCLUSIONES}

A lo largo del presente artículo se describió el funcionamiento de morfemas, llamados aquí "P", que pueden ocurrir superficialmente ligados al verbo o al nombre. Se mostró que esto se correlaciona con propiedades morfofonológicas de $\mathrm{P}$-esto es, si $\mathrm{P}$ o pro+P es mono o polisilábico, con excepciones en la segunda persona del plural-y con propiedades de su complemento, tanto sintácticas (si es "pesado" o "liviano") como semánticas (si es locativo o no, si es "familiar" o discursivamente accesible). Además, en aquellos casos en que el complemento de $\mathrm{P}$ ocurre a su derecha, $\mathrm{P}$ podría ser en realidad pro+P, donde pro es fonéticamente nulo, y su "complemento" sería un adjunto que duplica a pro; esto vale, por lo menos, para los PP polisilábicos.

Por otro lado, los aplicativos son mejor analizados como adposiciones que superficialmente se encliticizan al verbo y no como adposiciones incorporadas. Asimismo, se mostró que la oposición entre los complementos de aplicativo y los de adposición en chorote no se corresponde con la que existe típicamente entre argumentos aplicados y adjuntos, ya que los primeros no manifiestan las propiedades de un argumento aplicado canónico; más aún, éstos podrían analizarse incluso como adjuntos que doblan un complemento pronominal nulo.

Por último, existen casos en los que $\mathrm{P}$ no añade un argumento más al predicado, o al menos no uno explícito; éstos merecen mayor investigación.

\section{APÉNDICE}

A continuación se describen los principales procesos morfofonológicos que tienen lugar en chorote iyojwa'aja'.

(a) Bajo ciertas condiciones, detrás de vocales altas o glides subyacentes una consonante palataliza $(C \rightarrow C y)$. El proceso es morfofonológico y afecta especialmente a las coronales; para algunos detalles, véase Gerzenstein (1978).

(b) En frontera de morfema se inserta $y$ entre vocales: $/ \mathrm{i}-\mathrm{ho}^{\mathrm{h}}-\mathrm{y}^{\mathrm{h}} / \rightarrow / \mathrm{i}-\mathrm{h}$ ho-y-ey $/ \rightarrow$ ijyoyi 'va a'

(c) Una palatal cambia la altura de las vocales /a e/ siguientes en /e i/ respectivamente: a-tat-e 'arrojo', i-tyet-e 'arroja'; 'nes 'llega', ji-'nis 'llegás' (subyacentemente /hi'?nes/ $\rightarrow$ /hi'?n'1is/ (cf. (a) $\rightarrow$ [hi'?nis]). Sin embargo, algunas [a] superficiales (que considero $/ \alpha /$ subyacentes) no cambian: a-tal 'salgo', ji-tyal 'salís'.

(d) Las vocales altas /i u/ tienen alomorfos abiertos o laxos en posición tónica [ $\mathrm{\iota}$ v] 〈e ọ〉, a menos que preceda palatal(izada) o, en el caso de /u/, también /w/, p. ej. atojw [a'tuhw]

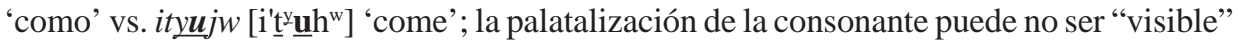
superficialmente, cf. ji-'nis (/hi'?n'y is/) ) 'llegás' en el parágrafo anterior.

(e) Las secuencias de sonante no silábica más laríngea subyacente sufren glotalización/ aspiración (o metátesis), p. ej. /'Tyan-hen/ (mirar-JEN) resulta 'yejnen 'observá'; /y-Pa'han-eh/ 
(3S-ser.sabio-INST) resulta 'yejéne. A su vez, las secuencias de oclusiva supralaríngea más /?/ producen una oclusiva glotalizada, p. ej. /t- $2 \alpha \mathrm{hl} /$ da $t$ ' $a l$ 'pide, ruega'; incluso /s- $1 /$ produce /ts'/: /'yiyis-?e/ da yiyits' $i$ ' 'sé'. Una laríngea sorda cae tras obstruyente, p. ej. /-tat-hen/ da -taten 'arrojar algo [a alguien]'.

(f) Las sonoras -incluyendo las vocales-en final absoluto son glotalizadas. Esto se indica con apóstrofo < '> antes de la consonante y después de la vocal, p. ej./y- $\alpha \mathrm{m} /$ ya'm/_\#\# 'se fue', /'y- $\alpha \mathrm{m}-\mathrm{a} / \rightarrow$ yama' /_\#\# 'se fue (PFv)'. En final de palabra /-h/ frecuentemente cae y no se representa ortográficamente, p. ej. (/s-?ahan-eh/ $\rightarrow$ ) /ts'a'haneh/ resulta ts'ajáne 'sé'; sin embargo, se conserva si ocurre en sílaba breve acentuada: /wuh/ resulta wuj 'muchos'. De este modo, una oposición subyacente / $\varnothing \sim \mathrm{h}$ / da lugar a una oposición superficial [? $~ \varnothing]$.

Los procesos de (f) se ignoran en la citación aislada de los morfemas: /-Re, -eh/ (LOC.P, INST) se citan -'e, - ej si bien en final de frase resultan -' $e$ ', $-e$. Sin embargo, para la citación de palabras completas se tienen en cuenta, p. ej. yi-'i ' 'está en', nam-e 'lo trae'.

(g) Las semivocales caen en coda (después de palatalizar la consonante siguiente cf. (a)): /hi-Pwen-ay/ '(ustedes) lo ven' resulta ji-'wen- $a$ en medio de frase y ji-'wen-a' (según (f)) en final absoluto.

(h) Después de vocal redondeada una laríngea puede labializarse, p. ej. /toh-eh/ da tojwe 'lejos'. Este proceso es léxico y no se aplica en todos los casos.

(i) Entre dos vocales separadas por laríngea, y cuando no ocurren (a) o (h), ocurren procesos de asimilación, tanto progresiva (a-tajl-e 'vengo de' vs. $\boldsymbol{a}$-tajl-aj-a 'venimos de') como regresiva ( $\varnothing$-'wanjl-e 'se queda' vs. $\varnothing$-'wanjl-a-jap' se queda junto a') cuyos detalles sería largo precisar. El último proceso es más “exterior” y ocurre opcionalmente entre palabras y clíticos.

\section{REFERENCIASBIBLIOGRÁFICAS}

AA. VV. (1997). Sinia' Jlamtis. El Nuevo Testamento en el idioma iyojwa'ja (chorote). Buenos Aires: Sociedad Bíblica Argentina.

AIKHENVALD, Alexandra (2000). Classifiers. A typology of noun categorization devices. Oxford: Oxford University Press.

BAKER, Mark (1988). Incorporation. A theory of Grammatical Function Changing. Chicago: University of Chicago Press. .(1996). The Polysynthesis Parameter. Oxford: Oxford University Press.

DOURADO, Luciana (2000). The advancement of obliques in Panara. In Santa Barbara Papers in Linguistics (Proceedings from the third Workshop on American Indigenous Languages), v. 10: 26-32.

.(2004). O avanço de oblíquos em Panará. In Wilmar D’Angelis (org.). Anais do II Encontro Macro-Jê. LIAMES 4: 43-50. Campinas: IEL/UNICAMP. 
DRAYSON, Nicholas (1999). Inamtis siuniwa awoki ti amtiyek'iji'n. 500 Palabras útiles en chorote. ASOCIANA. Tartagal.

.(2009). 'Niwak Samtis. Diccionario Iyojwa'ja 'Lij - Kilay 'Lij (Chorote-Castellano). In José Braunstein; Cristina Messineo (eds.). Hacia una nueva carta étnica del Gran Chaco VIII, pp. 91-174. Buenos Aires - Las Lomitas: PICT 32894 (ANPCyT), Centro del Hombre Antiguo Chaqueño.

DRAYSON, Nicholas; FRÍAS, Sebastián; GÓMEZ, Julián (2000). Sake' iyo ti iyojwa'jats'e'm. Somos chorotes - Nuestras costumbres. Tartagal: ASOCIANA.

FABRE, Alain (2010). El sufijo -sh del nivaclé (familia mataco-mataguayo) como instrumental, incremento de valencia y subordinador. In Lucía Golluscio; Alejandra Vidal (eds.). Las lenguas del Chaco. Estructura de la cláusula y relaciones interclausales. Amerindia 33/34: 43-72.

GERZENSTEIN, Ana (1978). Lengua chorote. Tomo 1. Buenos Aires: Universidad de Buenos Aires, FFyL, Instituto de Lingüística.

.(1979). Lengua chorote. Tomo 2. Buenos Aires: Universidad de Buenos Aires, FFyL, Instituto de Lingüística.

.(1983). Lengua chorote. Variedad 2. Buenos Aires: Universidad de Buenos Aires, FFyL, Instituto de Lingüística. .(1994). Lengua Maká. Estudio descriptivo. Buenos Aires: Universidad de Buenos Aires, FFyL, Instituto de Lingüística.

HALLE, Morris; MARANTZ, Alec (1993). Distributed Morphology and the Pieces of Inflection. In Kenneth Hale; Samuel Jay Keyser. The view from Building 20, pp. 111-176. Cambridge, Mass.: MIT Press.

JELINEK, Eloise (1984). Empty categories, case, and configurationality. Natural Language and Linguistic Theory 2: 39-76.

MARANTZ, Alec (1984). On the Nature of Grammatical Relations. Cambridge, Mass.: MIT Press.

MASULLO, Pascual (1992). Incorporation and the Case Theory in Spanish: a crosslinguistic perspective. Tesis doctoral. University of Washington.

PUJALTE, Mercedes (2009). Condiciones sobre la introducción de argumentos. La alternancia dativa en español. Tesis de maestría. Escuela Superior de Idiomas, Universidad Nacional del Comahue.

PYLKKÄNEN, Liina (2002). Introducing Arguments. Tesis doctoral. Cambridge, Mass.: MIT Press.

Recebido: $12 / 3 / 2011$

Versão revista: 8/6/2011

Aceito: 20/6/2011. 